\title{
10 ANÁLISIS DE UNA EXPERIENCIA DE ESTIMULACIÓN MULTISENSORIAL CON EL MÉTODO ESTITSOLÓGICO
}

\author{
Dra. Pilar Ibáñez
}

Profesora Titular del Departamento MIDE

Facultad de Educación. UNED

Dra. María Senra

Profesora Colaboradora del Departamento MIDE Facultad de Educación. UNED

\section{IINTRODUCCIÓN}

Después de varios años trabajando con poblaciones especiales e investigando con personas que no están integradas en los medios habituales de vida, han centrado nuestra atención y preocupación las madres que están en prisión con sus hijos, porque pensamos que hasta el momento esa población no ha sido suficientemente estudiada, sobre todo en el aspecto afectivo.

En las sentencias a delincuentes, frecuentemente se suele reflejar la carencia afectiva que han sufrido desde niños. Sabemos y reconocemos que, si las personas han sido queridas en su infancia y se les ha demostrado escuchándolas, acariciándolas y estableciendo con ellas contacto visual, se sienten seguras y se relacionan bien con las demás personas. En caso contrario, con frecuencia muestran temores, desconfianza, incomprensión, celos y otros rasgos de carácter que las sitúan entre las personas llamadas difíciles.

Por supuesto, no todas las personas que han tenido infancias difíciles son adultos marginales, porque suelen poner en marcha mecanismos de defensa o evasión hacia contextos sociales normalizados. 
Este trabajo surgió por el deseo de conocer los contextos afectivos de las madres encarceladas y afianzar las relaciones o nexos afectivos con sus hijos, a fin de que en su infancia, si es preciso, no carezcan de lo que todo niño debería tener, sea cualesquiera el modus vivendi familiar. Nuestro objetivo inmediato es ayudar a esas madres y sus bebés para que sus relaciones afectivas se incrementen, potenciando sus vínculos afectivos mediante el método estitsológico de estimulación multisensorial para compensar las posibles carencias debidas al medio ecológico adverso en que se desarrolla su vida.

\section{| PLANTEAMiento DEL PROBLEMA}

Los conflictos generacionales están en auge este fin de siglo. Lo que antes era una excepción se está convirtiendo en regla: peleas en el hogar, incomunicación padres-hijos e incluso malos tratos. Los medios de comunicación han tomado parte en la batalla generacional con el ya popular slogan: "habla con tu hijo". Reconocen así la importancia y necesidad de la comunicación desde la infancia como vacuna contra la alienación padres-hijos.

La preocupación por el tema traspasa nuestras fronteras, especialmente en países desarrollados; como muestra nos referiremos a los trabajos presentados en la Conferencia de Washington (1997) en los que se indicaba que el 25\% de los padres desconoce que su comportamiento afectivo puede influir en el desarrollo de la inteligencia de su hijo (Goleman, 1993; Jáuregui, 1994) y, por supuesto, en la configuración de su personalidad. Tan sólo de un $20 \%$ a un $30 \%$ les estimula instintivamente en el momento adecuado.

Que los niños necesitan estar, al menos algún tiempo, diariamente con su madre/padre no requiere comentario. Son muchos los autores que se han ocupado del estudio de las relaciones afectivas entre madre-hijo, entre ellos cabe destacar como una de las figuras pioneras a Melanie Klein (1937), así como a Brazelton (1974), Spita (1979), Wolf (1965), etc.

Las relaciones afectivas se pueden establecer desde antes del nacimiento del bebé, ya que durante la gestación se establecen las líneas principales de los circuitos que controlan las emociones. Parece ser que éstas inciden incluso en su nacimiento (Florez-Lozano, 1980). Posteriormente la actuación de los padres es decisiva.

Es sabido que el feto influye en los cambios fisiológicos y psicológicos de la madre, y ésta, a su vez, consciente o inconscientemente, también actúa sobre él, como muestran los numerosos estudios experimentales; de ahí la gran importancia que tiene la actitud de la futura madre hacia su hijo. 
Es igualmente conocida la importancia de establecer una relación con el hijo, aun antes de nacer, hablándole, acariciándose el vientre, escuchando música juntos, tranquilizándole y transmitiéndole los sentimientos de aceptación y el estado de ánimo del que es portadora la madre; por ello hay que prestar especial atención a la tranquilidad de la gestante y a las estimulaciones que van a intervenir en el desarrollo sensorial y mental.

La estimulación tanto de humanos como de animales es tan antigua como el comienzo de los tiempos, mas su permanencia y aplicación sistemática sólo se ha conservado en ciertas culturas, siendo el tacto uno de los sentidos al que se le ha dado mayor importancia en las primeras comunicaciones entre padres e hijos (Salvatierra, 1983).

La evolución del sistema sensorial y .motriz va a quedar reflejada en el cociente de desarrollo. Pero no sólo se va a producir un aumento de inteligencia, en terminología común, sino una mayor unión y comprensión entre padres e hijos (Ibáñez, P., 1997).

Las investigaciones sobre el cerebro no cesan, pero nadie duda de la importancia de la niñez para el establecimiento de las conexiones cerebrales por medio de la actividad tanto sensorial como cognitiva (Barbizet, 1978), y se indican los tres primeros años de vida como muy importantes para las conformaciones que tanto han de incidir a lo largo de la vida del sujeto.

Según Spitz (1979), el periodo crítico en el que se puede producir un considerable retraso intelectual está representado por los ocho primeros meses, pero esta peligrosidad persiste hasta los cuatro años aproximadamente. Después de esta edad la privación suele manifestarse con la aparición de rasgos caracteriales más o menos severos.

\section{EL MÉTODO ESTITSOLÓGICo}

La estitsología estudia la influencia de los estímulos en el proceso de maduración del sistema nervioso del niño.

Las primeras vivencias del recién nacido surgen a través de sus sentidos corporales y son facilitadas por el contacto afectivo que le proporcionan sus padres. En el niño se van produciendo unas pautas de conducta que le permiten adaptarse a su entorno y que van a marcar sus respuestas conductuales en el futuro.

El proceso natural de maduración ha de ser estimulado hacia metas superiores específicamente humanas. Compartimos con Goleman (1993) la gran importancia de la afectividad para configurar el desarrollo de individuos 
emocionalmente inteligentes y socialmente integrados. En esta línea se sientan las bases del Método Estitsológico de estimulación multisensorial cuya finalidad general es la estimulación del niño tanto sensorial como afectiva y el desarrollo de los lazos afectivos entre madre e hijo.

Su técnica básica es la estimulación multisensorial adecuada (Ibáñez, 1997). Sus objetivos son:

a) Para el niño:

- Afianzar los nexos afectivos con la madre/padre que le aplica el método.

- Estimular los sistemas nervioso y muscular a través de la sensibilidad cutánea.

- Intervenir en la evolución de los procesos cognitivos a través de la estimulación multisensorial y motriz.

b) Para los padres:

- Liberarse de preocupaciones y compartir un momento de relajación e intimidad con su hijo.

- Aprender a interpretar el lenguaje corporal de su hijo

- Adquirir autoconfianza en el trato con el niño

- Liberarse del sentimiento de abandono al hijo cuando se le ve poco.

Estos objetivos se persiguen mediante una serie de actividades sistemáticamente desarrolladas y adecuadas a cada edad desde el nacimiento.

Es fundamental concienciarse de que la/el madre/padre es más importante que cualquier juguete, por ello es muy importante optimizar la calidad del tiempo compartido.

\section{METODOLOGÍA}

La realización de este trabajo se llevó a cabo con un estudio longitudinal con un grupo de mujeres encarceladas, madres de bebés nacidos en la cárcel y que compartían con ellas el mismo espacio vital.

Empleamos el procedimiento del test retest, es decir, le aplicamos los test antes de iniciar el proceso de aplicación de la estimulación y se volvió a hacer otra observación y aplicación después de nueve meses de estimulación con el Método Estitsológico. 
Hemos realizado entrevistas individuales con cada madre y para la observación de su hijo.

El estudio, por razones obvias, es anónimo, aunque con fines identificativos y asociativos para la segunda aplicación hemos seguido el sistema de numeración.

\section{a) Muestra}

La muestra inicial estaba formada por 25 mujeres con sus respectivos bebés, pero, por su propia configuración, es una población inestable y en la segunda aplicación solamente hemos encontrado nueve mujeres de las que formaban la muestra inicial; por lo tanto, éstas forman la muestra de estudio, debiendo desechar las once restantes porque no pudimos realizar su seguimiento.

Los niños de la muestra inicialmente eran 8 varones y 17 mujeres, con una media de edad de 5 meses, pero nuestra muestra final quedó constituida por 2 varones y 7 mujeres, con una media de edad al finalizar el estudio de 14 meses.

\section{b) Instrumento}

Para la anamnesis con la madre hemos utilizado un cuestionario semiabierto mediante entrevista, que nos ha facilitado los datos relacionados con su infancia y adolescencia, embarazo, parto, etc., así como los datos referidos a su hijo.

Para el niño utilizamos el test de Gessel que aplicamos en test y retest con el fin de observar la evolución de su desarrollo después de nueve meses de estimulación multisensorial con el Método Estitsológico. Las actividades de estimulación fueron realizadas por las madres, a las que previamente se les enseñaba y se las instruía en su aplicación y seguimiento, así como su utilidad y finalidad.

\section{c) Procedimiento}

Nuestro proyecto de trabajo fue muy bien acogido por la dirección de la Penitenciaría de Carabanchel para desarrollarlo con madres y sus bebés. Agradecemos desde aquí su colaboración y las facilidades que nos han brindado.

Para la explicación y aprendizaje del Método Estitsológico, las reclusas asistían voluntariamente a unas sesiones en las que se desarrollaban actividades de formación y prácticas de entrenamiento. Es necesario señalar que las reclusas, entre sus obligaciones, tienen la de atender a sus hijos y 
cuidar de su higiene y limpieza; por estos actos se le suele aplicar reducción de condena.

A pesar de ser voluntaria la asistencia a las sesiones, hemos de reflejar algunas características contextuales, y decimos contextuales porque lo mismo sucedía en otras actividades dirigidas por personas no pertenecientes a la penitenciaría.

En términos generales, el control de tiempo parece no existir para las reclusas, a pesar de haberse fijado el horario de asistencia y ser el mismo durante todo el tiempo que las visitamos. Las sesiones siempre comenzaban tarde, porque algunas no estaban preparadas o tenían que ausentarse por más de un motivo; además su asistencia era irregular, iban un día y al siguiente no lo hacían, presentándose nuevamente el tercer día o incluso cuando se estaba trabajando con otro grupo, lo que dificultaba la enseñanza de un método secuenciado.

No obstante, como estamos acostumbradas a trabajar con poblaciones especiales, realizamos nuestro proceso de adaptación y los resultados no tardaron en ser satisfactorios.

La entrevista al principio les producía temor, hecho que comprendemos dada su situación y las numerosas entrevistas e interrogatorios a que han sido sometidas, pero pronto fueron accesibles, como muestran los cuestionarios y pruebas que les aplicamos.

Respecto a su situación como internas con las que trabajamos, apreciamos que, a pesar de sus deseos de salir de allí, se habían adaptado a la situación, sus hijos eran para ellas un modo de evasión que les hacía tener su tiempo ocupado. El jardín de que disponían les permitía pasear o pasar su buen tiempo al aire libre con sus hijos. Su información del mundo exterior se realizaba especialmente a través de la televisión, aunque recibían visitas de organizaciones de voluntariado y de familiares y amigos.

Se nos dijo que gran número de madres en la penitenciaría no sabían jugar con sus hijos, por lo tanto la emisión de estímulos quedaba disminuida, ya que, en los primeros meses del bebé, es la madre la única persona que se ocupa del niño, hasta que éste comienza su asistencia a la guardería del centro penitenciario y allí se relaciona con los otros niños de madres reclusas.

\section{RESULTADOS}

Los resultados obtenidos los referimos como punto de partida inicial a los datos descriptivos de la población de referencia, es decir, la muestra global de 25 casos que inicialmente habíamos recogido. 


\section{a) Muestra inicial:}

Encontramos que el $60 \%$ de las madres son de nacionalidad española y el resto son hispanoamericanas y africanas. La mayoría tenían pareja fija al quedar embarazadas $(76 \%)$ y la han seguido manteniendo, aunque un $8 \%$ fueron abandonadas por su pareja al ingresar en prisión.

El $68 \%$ de los padres de los niños están en libertad y un $44 \%$ tienen trabajo.

b) Muestra final y definitiva:

Limitándonos a la muestra concreta de nueve madres con sus hijos que siguieron el proceso de estimulación y continuaban en la cárcel al finalizar el mismo después de 9 meses, los resultados que obtuvimos son los siguientes:

La mayoría de las madres $(77,7 \%)$ han tenido un parto sin problemas. El $22 \%$ de los niños nació con síndrome de abstinencia y el $11 \%$ con anticuerpos. Su peso medio al nacer ha sido de $3 \mathrm{kgrs}$. La alimentación recibida por los niños se distribuye por igual $(33,3 \%)$ entre alimentación natural, artificial y mixta.

Debido a la condición de presidiaria de las madres, consideramos de gran interés para nuestros objetivos conocer la actitud de la madre ante su embarazo, por la posible influencia o interacción en su relación afectiva con el hijo.

Hemos encontrado un $66,6 \%$ de madres que manifiesta que su embarazo fue buscado, mientras que el $33,3 \%$ restante dice que fue accidental. Lo absurdo que puede parecer el buscar un embarazo en su situación tiene su explicación en el hecho de que las madres con bebés reciben un trato especial y distinto al de las otras mujeres encarceladas. Su única obligación es cuidar al niño hasta que vaya a la guardería y, además, dicen que con el hijo se sienten acompañadas.

Cuando las madres se enteraron de su embarazo, la mayoría sintieron alegría $(66,6 \%)$ y el restante porcentaje manifiesta que sintió preocupación ante la situación que debía afrontar.

La mayoría de las madres $(77,7 \%)$ manifiesta que tomaba a su hijo en brazos cuando lloraba e incluso un 55\% dice que le gustaba tenerlo en brazos sin ningún motivo, simplemente porque se sentía madre.

Con la Escala de Desarrollo de Gessel obtuvimos los siguientes datos referidos al cociente de desarrollo general: 


\section{TABIA I}

Exolución del cociente de desarrollo general

\begin{tabular}{|c|c|c|c|}
\hline Casos & C.D. antes & C.D. después & Evolución \\
\hline 1 & 100 & 100 & 0 \\
2 & 125 & 130 & 5 \\
3 & 105 & 111 & 6 \\
4 & 89 & 90 & 1 \\
5 & 96 & 98 & 2 \\
6 & 103 & 106 & 3 \\
7 & 95 & 97 & 2 \\
8 & 101 & 104 & 3 \\
9 & 100 & 102 & 2 \\
Media & 101 & 104 & 2,67 \\
\hline
\end{tabular}

Respecto a los distintos factores de desarrollo que se obtienen con la Escala de Desarrollo de Gessel, podemos ver la distribución de medias, así como la evolución de cada uno de ellos, en la siguiente tabla:

TABI A II

Distribución de medias de factores de desarrollo

\begin{tabular}{|c|c|c|c|}
\hline C. de Desarrollo & Antes & Después & Evolución \\
\hline General & 101 & 104 & 3 \\
Motriz & 103,6 & 106,3 & 2,7 \\
Adaptativo & 96,8 & 100,5 & 3,7 \\
Lenguaje & 100,4 & 104,3 & 3,9 \\
Social & 105 & 105,6 & 0,6 \\
\hline
\end{tabular}

Como podemos observar, la media de todos los factores de desarrollo ha evolucionado después de los 9 meses de estimulación multisensorial. Al someterlos a correlación, todos correlacionaban positivamente.

Con el fín de conocer la significación estadística de estos datos, los hemos analizado con la prueba de la "t pareada", ya que es una prueba estadística específica que permite analizar la homogeneidad de dos medias en datos pareados (Carrasco, 1982). 
Los resultados obtenidos son los siguientes:

\section{Estimulación y Cociente General de Desarrollo}

La comparación de medias del C.D. General obtenido con la Escala de Desarrollo de Gessel, antes y después del periodo de estimulación, arroja una diferencia significativa para $P<0,01$, lo cual nos permite afirmar que la evolución del C.D. General entre una y otra aplicación es significativa y no se debe al azar.

\section{Estimulación y Factores de Desarrollo}

Al someter a contraste las medias de los diversos factores de desarrollo obtenidos con la escala de Gessel, encontramos que las diferencias de medias de ambas aplicaciones en desarrollo psicomotriz, adaptativo y lenguaje son estadísticamente significativas. No hay significación en la diferencia de medias en el factor social obtenida en las dos aplicaciones de la escala de Gessel.

Estos datos nos permiten afirmar que la evolución en cociente de desarrollo general y en los factores motriz, adaptativo y de lenguaje, después de un periodo de estimulación multisensorial de nueve meses, es significativa y no se debe al azar.

\section{Embarazo y Cociente de Desarrollo}

Hemos sometido también a contraste estadístico el cociente de desarrollo antes y después de la aplicación del periodo de estimulación multisensorial, con el hecho de que la madre tuviese un embarazo buscado o no y con la actitud de la madre al saberse embarazada, con el fin de analizar la posible relación de estos factores con la evolución del desarrollo del niño.

Con los resultados obtenidos podemos afirmar que la evolución del C.D. general es estadísticamente significativa $(P<0,05)$ en aquellos niños cuyo embarazo había sido buscado y deseado, mientras que no ocurre así en los niños cuyo embarazo había sido accidental.

\section{Embarazo y Factores de Desarrollo}

Es igualmente significativa $(\mathrm{P}<0,01)$ la evolución del desarrollo motriz en los niños de embarazo buscado y no es significativo en los de embarazo accidental. 
La evolución del desarrollo del lenguaje es igualmente significativa $(P<0,05)$ en los niños cuyo embarazo había sido buscado y carece de significación en aquellos cuyo embarazo fue accidental.

\section{Actitud de la madre ante el embarazo y Cociente de Desarrollo}

En el contraste del cociente de desarrollo general con la actitud de alegría o preocupación de la madre ante el embarazo, encontramos que es estadísticamente significativa con un $P<0,05$ la evolución del C.D. general de aquellos niños cuya madre sintió alegría al saberse embarazada y no hay significación en la evolución de aquellos niños cuya madre sintió preocupación.

\section{Actitud de la madre ante el embarazo y Factores de Desarrollo}

$\mathrm{Al}$ analizar los factores de desarrollo encontramos igualmente significación $(P<0,05)$ en el desarrollo motriz de los niños cuya madre sintió alegría al saberse embarazada, mientras que no hay significación en los niños cuya madre sintió preocupación.

En los demás factores de desarrollo no hay significación en relación con esta variable.

\section{CONCLUSIÓN PROVISIONAL}

Es necesario señalar en primer lugar que este análisis de una experiencia de estimulación multisensorial con el Método Estitsológico es solamente una toma de contacto con lo que nos hemos propuesto como una amplia investigación, ya en marcha, con poblaciones especiales.

No pretendemos que los resultados obtenidos sean categóricos, dada la limitación de la muestra, pero era un paso necesario porque confiamos en la eficacia del Método Estitsológico en poblaciones especiales y consideramos que los resultados obtenidos, aunque modestos, son suficientemente representativos para permitirnos seguir en esta línea de investigación con muestras más amplias de distintas poblaciones especiales.

La evolución del desarrollo general del niño después de la estimulación multisensorial parece un hecho evidente, pero en ulteriores investigaciones en esta línea estaremos en disposición de confirmar estos resultados que pueden ser una esperanza para niños de poblaciones desfavorecidas. 


\section{BIBLIOGRAFÍA}

BARBIZET, J. y DUIZABO, Ph. (1977). Manual de Neuropsicología. Barcelona. Toray-Masson.

BRAZELTON, T. B. (1974). The origins of reciprocity: the early mother infant interaction. En Lewis, M.: The effects of the infant on its caregiver. New york. Silley.

CARRASCO, J. L. (1982). El Método Estadístico en la investigación médica. Madrid, Karpos, S. A.

FLOREZ-LOZANO, J. A. (1980). Incidencia de algunos factores psicofisiológicos en el desarrollo físico y psíquico de niñas internas en un orfanato. Acta Pediátrica, n. 442.

GESSEL, A. y AMATRUDA, C. (1986). Diagnóstico sobre el desarrollo normal $y$ anormal del niño. Buenos Aires, Paidós.
GOLEMAN, D. (1993). Inteligencia emocional. Barcelona. Kairós.

IBÁÑEZ LÓPEZ, P. (1997). Aumente la inteligencia de su hijo. Madrid, Dykinson.

JÁUREGUI, A. (1994). El ordenador cerebral. Madrid, Labor.

KLEIN, M. (1937). Love, hate and reparation. London. Hogarth Press.

SALVATIERRA BAEZA, M. R. (1985). Ensayo y experimentación para la aproximación a una nueva stitsología. Tesis inédita. Universidad Complutense. Madrid.

SPITZ, R. A. (1979). El primer año de vida del niño Madrid, Aguilar.

WOLF, P. (1965). The causes, controls and organization of behavior in the neonate. Psychological Issues (Monograph 17), Vol. 5.

\section{RESUMEN}

Análisis de una experiencia de estimulación multisensorial con hijos (bebés) de mujeres recluidas en el centro penitenciario de Carabanchel.

Durante un periodo de nueve meses se aplicó a los niños un proceso sistemático de estimulación multisensorial dirigido a compensar las carencias derivadas del ambiente desfavorecido en el que se desarrollan.

En este proceso de estimulación multisensorial utilizamos el Método Estitsologico Multisensorial Elemental.

Se realizó una evaluación inicial de su desarrollo con el test Gessel y, una vez transcurrido el periodo de estimulación previamente establecido, se realizó una nueva evaluación de su desarrollo general y factorial

Finalmente se someten los datos a un análisis estadístico de comparación de medias.

Palabras clave: Estimulación temprana multisensorial, Prisión: madres y bebés, Estudio longitudinal evolutivo del desarrollo, Método Estitsológico. 


\section{ABSTRACT}

Analysis of a multisensorial experience with babies of imprisoned women at the penitentiary centre of Carabanchel (Madrid. Spain).

During a period of nine months we applied to children a systematic process of stimulation aimed to compensate the lacks derived from the special situation in which they develop.

In this process of multisensorial stimulation we employed the Estitsologic Multisensorial Method.

An initial evaluation of their development was carried out with the Gessel scale and, once the period of stimulation previously established was over, a new evaluation of their general and factorial development was made.

Finally, all data was assesed through statistical analysis.

Key words: Early Multisensorial Stimulation, prison: mothers and babies, longitudinal evolutive study of development, Estitsological Method. 